\title{
Preoperative risk factors for extraurothelial recurrence in NOMO patients with renal pelvic cancer treated by radical nephroureterectomy
}

\author{
KEIICHI ITO, JUNICHI ASAKUMA, KENJI KURODA, KAZUYOSHI TACHI, \\ AKINORI SATO, AKIO HORIGUCHI, KENJI SEGUCHI and TOMOHIKO ASANO \\ Department of Urology, National Defense Medical College, Tokorozawa, Saitama 359-8513, Japan
}

Received August 5, 2015; Accepted January 22, 2016

DOI: $10.3892 / \mathrm{mco} .2016 .754$

\begin{abstract}
The prediction of extraurothelial recurrence (EUR) before radical nephroureterectomy (RNU) is difficult for patients with upper tract urothelial carcinoma. If EUR can be predicted preoperatively, it may be possible to determine the need for neoadjuvant chemotherapy or the surgical strategy. Our previous study identified preoperative risk factors for EUR in ureteral cancer, while the present study identified preoperative risk factors for EUR in renal pelvic cancer (RPC). The preoperative factors were reviewed in 71 NOM0 patients with RPC treated by RNU between 1999 and 2013. Preoperative clinical background data, laboratory test results, including inflammatory indices, lactate dehydrogenase (LDH), and estimated glomerular filtration rate, and radiological findings were subjected to multivariate analyses to identify independent predictive factors for EUR. The 3-year EUR-free survival (EURFS) rate was $81.9 \%$, and univariate analysis showed that clinical T (cT) stage $\geq 3$, white blood cell counts $\geq 7600 / \mu 1$, neutrophil counts $\geq 4,500 / \mu 1$, neutrophil-to-lymphocyte ratio $\geq 2.0$, and $\mathrm{LDH} \geq 210 \mathrm{IU} / 1$ were significantly associated with EURFS. Additionally, a cT stage $\geq 3(\mathrm{P}=0.0244)$ and $\mathrm{LDH}$ $\geq 210 \mathrm{IU} / 1$ ( $\mathrm{P}=0.0322$ ) were independent predictors for EUR. When patients were stratified into three groups according to the number of risk factors, the 3-year EURFS rates were 94.5, 76.3, and 33.3\% for the low-, intermediate-, and high-risk patient groups, respectively. In conclusion, cT stage $\geq 3$ and $\mathrm{LDH} \geq 210$ IU/l were preoperative predictive factors of EUR in patients with RPC. High-risk patients may be candidates for neoadjuvant chemotherapy and low-risk patients may be candidates for RNU without lymph node dissection.
\end{abstract}

Correspondence to: Dr Keiichi Ito, Department of Urology, National Defense Medical College, 3-2 Namiki, Tokorozawa, Saitama 359-8513, Japan

E-mail: itok@ndmc.ac.jp

Key words: extraurothelial recurrence, lactate dehydrogenase, nephroureterectomy, preoperative factor, renal pelvic cancer

\section{Introduction}

Extraurothelial recurrence (EUR) is difficult to predict in patients with upper tract urothelial carcinoma (UTUC) prior to radical nephroureterectomy (RNU). Previously reported predictors for EUR, such as pathological T (pT) stage and lymphovascular invasion (LVI) (1-4), cannot be determined prior to surgery. Identification of high-risk patients for EUR using preoperative factors, may allow neoadjuvant chemotherapy followed by RNU with extended lymph node dissection (ELND) to improve the survival of these patients. By contrast, determining which patients have a low-risk for EUR, may allow minimally-invasive surgery such as laparoscopic RNU without lymph node dissection (LND) to be used safely in these individuals.

A previous study was performed to identify the preoperative predictors for recurrence in UTUC patients, and clinical $\mathrm{T}$ stage and a neutrophil counts of $\geq 4,000 / \mu 1$ were independent predictors of recurrence (5). Additional previous studies (6-8) have tried to identify the preoperative predictors of muscle-invasive or non-organ-confined disease, as patients with these conditions are considered to be at very high risk of UTUC recurrence. In these previous reports, tumor location, cytology, the presence of hydronephrosis, local invasion observed on imaging, a high-grade tumor confirmed by ureteroscopic biopsy, architecture observed on ureteroscopy were identified for predictors for muscle-invasive or non-organ-confined disease in UTUC patients (6-8). However, the authors performed ureteroscopy in all patients. Routine use of ureteroscopy before RNU appeared to be an invasive procedure for some UTUC patients. Predicting EUR by using more simple preoperative factors would reduce the invasiveness of the diagnostic methods for the patients.

Due to anatomical differences, it did not appear to be appropriate to categorize preoperative radiological findings of renal pelvic cancer (RPC) and those of ureteral cancer (UTC) using the same classification criteria. For example, RPC located at renal calyx is unlikely to invade the Gerota's fascia because of the presence of renal parenchyma. By contrast, UTC easily invades the surrounding tissues. Furthermore, invasive UTC easily leads to the full dilation of the renal pelvis and renal calyces, whereas RPC does not easily lead to the full dilation 
of the renal pelvis and renal calyces, even when RPC invades the renal parenchyma. In addition, some authors reported that patients with UTC had a worse prognosis than that of patients with RPC, when tumor grades or stages are comparable $(9,10)$. Therefore, patients with RPC and UTC were analyzed separately, with a focus on patients with RPC.

We previously analyzed patients with UTC to determine preoperative risk factors for EUR and demonstrated that clinical T (cT) stage, ureteral tumor length along the ureter $\geq 3 \mathrm{~cm}$, positive urine cytology, and an estimated glomerular filtration rate $(\mathrm{eGFR})<60 \mu \mathrm{l} / \mathrm{min} / 1.73 \mathrm{~m}^{2}$ were independent predictors for EUR, and risk classification for EUR could be established in patients with UTC (11). Furthermore, patients in the high-risk group rapidly developed recurrence, and therefore appeared to be potential candidates for neoadjuvant chemotherapy. In addition, patients in the low-risk group had a low risk for EUR, and appeared to be candidates for omission of LND.

The aim of the present study was to identify preoperative predictors for EUR in patients with RPC.

\section{Patients and methods}

The medical records of 150 N0M0 patients with pathologically diagnosed urothelial carcinoma, and treated by unilateral RNU at our institute between April 1999 and July 2013 were retrospectively reviewed. Of the 150 patients, 76 had RPC, including one with concomitant invasive bladder cancer who underwent both RNU and radical cystectomy, and another with RPC who underwent radical cystectomy 1 year prior to RNU, due to muscle invasive bladder cancer. Therefore, these two patients were excluded from the present study. Three patients with concomitant UTC with radiologically confirmed invasive growth were also excluded from the present study. Finally, a total of 71 patients with RPC were evaluated in the present study. Among these patients, noninvasive UTC was suspected in three according to preoperative radiological examinations. The median follow-up period was 50.3 months (range, 1-160 months). The study protocol was approved by the institutional review board (approval no. 2086).

Forty patients underwent open nephroureterectomy, while 31 were treated by laparoscopic nephroureterectomy. Regional LND was performed in 13 patients $(18.3 \%)$ with suspected enlarged LNs, detected during intraoperative inspection, or with suspected advanced clinical stage by preoperative radiological examinations. The extent of regional LND was often limited (e.g., only the renal hilus) and ELND was not routinely performed. One of the 13 patients who underwent LND was found to have LNM. Of the remaining 12 patients with pNO disease, recurrence developed in two (lymph node metastasis revealed in 1 of the 2 patients). No patients in the present study received neoadjuvant chemotherapy. Cisplatin-based adjuvant chemotherapy was administered to 12 patients with pathologically confirmed lymph node metastasis or muscle invasive disease. Local recurrence and metastasis were monitored by examining each patient every 3-6 months for the first 5 years following surgery, and every 6-12 months thereafter. Intravesical recurrence and recurrence in the contralateral upper urinary tract (UUT) were not considered as EUR in the current study. No patient developed recurrence in the contralateral UUT.
Radiological findings, including cT stage, presence of hydrocalyx, and maximal tumor size on an axial view were determined by computed tomography (CT) and/or magnetic resonance imaging (MRI). Clinical $\mathrm{T}$ stage was determined according to the TNM classification (12) and classified as $\mathrm{T} \leq 2$ or $\geq 3$. Contrast-enhanced CT and/or contrast-enhanced MRI were used for the determination of cT stage. If RPC patients had decreased renal function, both CT and MRI without contrast media were used. The lesion was considered to be a clinical T3 tumor when the tumor obviously extended into the adjacent renal parenchyma or when the margin of the tumor was irregular and the invasion toward peripelvic fat tissue was strongly suspected. The lesion without obvious invasion toward renal parenchyma or peripelvic fat tissue was considered to be clinical $\mathrm{T} 2$ or less.

Urine cytology was evaluated by Papanicolaou staining. Positive cytology was defined as the presence of malignant or atypical cells in voided specimens. Grading of Papanicolaou staining was determined using a 5-grade system. Inflammatory indices (white blood cell count, neutrophil count, C-reactive protein level, and neutrophil-to-lymphocyte ratio) (13) were evaluated using laboratory tests. The neutrophil-to-lymphocyte ratio (NLR) was calculated by dividing the absolute neutrophil count by the absolute lymphocyte count. The eGFR was calculated using the following equation: eGFR $\left(\mathrm{ml} / \mathrm{min} / 1.73 \mathrm{~m}^{2}\right)=194 \times(0.739$, if female) $\times$ (serum creatinine $)^{-1.094} \mathrm{x}$ age $\mathrm{e}^{-0.287}$. The cut-off values for these laboratory tests are shown in Table I. Upper normal limits were used as cut-off values for C-reactive protein (CRP) levels. For white blood cell (WBC) count, neutrophil count, NLR, and lactate dehydrogenase ( $\mathrm{LDH})$, the values best discriminating between good and poor survival was determined by testing all possible cut-off values within the central $85 \%$ of the distribution of values. Laboratory data were obtained by performing blood tests within one month prior to RNU.

Statistical analysis. Results are presented as means \pm standard deviations. Survival curves were constructed using the Kaplan-Meier method, and differences between them were assessed using the log-rank test. To identify preoperative predictors of EUR, univariate and multivariate analyses were performed using the Cox proportional hazards model. $\mathrm{P}<0.05$ was considered to indicate a statistically significant difference.

\section{Results}

Patient characteristics, radiological findings, and laboratory test results are listed in Table I. Twelve (16.9\%) of 71 patients were diagnosed with EUR. Of these 12 patients, initial recurrence developed in the LNs in seven, the distant organs in nine, and both LNs and distant organs in four. The median time to EUR was 6.5 months (range, 1.0-13.0) in the 12 patients.

The 3-year EUR-free survival (EURFS) rate was $81.9 \%$ (Fig. 1). The EURFS rates (Fig. 2) were significantly lower in patients with $\mathrm{cT} \geq 3$ than in those with $\mathrm{cT} \leq 2$ (Fig. $2 \mathrm{~A}$ ), patients with neutrophil counts $\geq 4,500 / \mu$ l than in those with neutrophil counts $<4,500 / \mu$ l (Fig. 2 B), patients with NLR $\geq 2.0$ than in those with NLR $<2.0$ (Fig. 2 C), patients with $\mathrm{LDH} \geq 210 \mathrm{IU} / 1$ than in those with LDH $<210$ IU/1 (Fig. 2D), and patients 
Table I. Preoperative variables in NOM0 patients with renal pelvic cancer $(n=71)$.

\begin{tabular}{|c|c|c|}
\hline Variables & Categories & No. of patients \\
\hline Age (years) & $\geq 65$ vs. $<65$ & $51 / 20$ \\
\hline Gender & Male vs. Female & $58 / 13$ \\
\hline Tumor side & Right vs. Left & $32 / 39$ \\
\hline Past history of BT and/or concomitant BT & Yes vs. No & $18 / 53$ \\
\hline Symptomatic & Yes vs. No & $50 / 21$ \\
\hline Concomitant ureteral lesion (radiological) & Presence vs. Absence & $5 / 66$ \\
\hline Hydrocaryx (at least 1 calyx) & Presence vs. Absence & $26 / 45$ \\
\hline Clinical T stage & $\mathrm{cT} \leq 2$ vs. $\mathrm{cT} \geq 3$ & $52 / 19$ \\
\hline \multirow[t]{4}{*}{ Maximal tumor size ${ }^{\mathrm{a}}(\mathrm{cm})$} & $<1.5$ & 8 \\
\hline & $1.5-3.0$ & 28 \\
\hline & $3.0-4.0$ & 21 \\
\hline & $\geq 4.0$ & 14 \\
\hline Urine cytology & Positive vs. Negative (ND ${ }^{\mathrm{b}}$ ) & $51 / 19(1)$ \\
\hline WBC count $(/ \mu 1)$ & $>7600$ vs. $\leq 7600$ & $64 / 7$ \\
\hline Neutrophil count $(/ \mu 1)$ & $>4,500$ vs. $\leq 4,500$ & $58 / 13$ \\
\hline $\mathrm{CRP}(\mathrm{mg} / \mathrm{dl})$ & $>0.3$ vs. $\leq 0.3$ & $54 / 17$ \\
\hline NLR & $>2.0$ vs. $\leq 2.0$ & $41 / 30$ \\
\hline LDH (IU/l) & $>210$ vs. $\leq 210$ & $52 / 19$ \\
\hline eGFR (ml/min./1.73 $\left.\mathrm{m}^{2}\right)$ & $>60$ vs. $\leq 60$ & $27 / 44$ \\
\hline
\end{tabular}

NOM0, node 0, metastasis 0; BT, bladder tumor; CRP, C-reactive protein; eGFR, estimated glomerular filtration ratio; LDH, lactate dehydrogenase; ND, not determined; NLR, neutrophil-to-lymphocyte rate. ${ }^{a}$ Maximal tumor size was measured in axial view of CT or MRI. ${ }^{b}$ Not determined due to the patient undergoing hemodialysis.

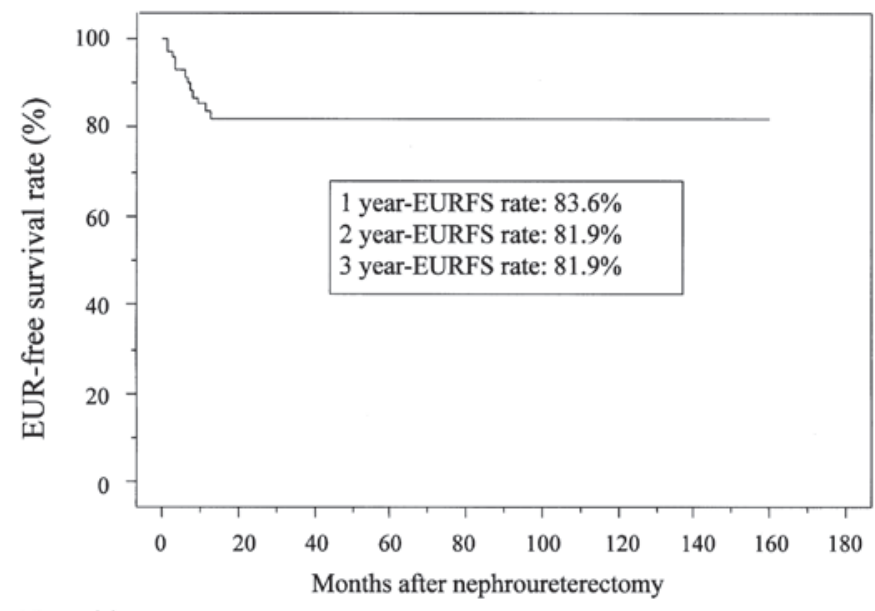

No. at risk

$\begin{array}{llllllllll}71 & 49 & 41 & 30 & 18 & 9 & 4 & 2 & 1 & 0\end{array}$

Figure 1. Extraurothelial recurrence-free survival rates following radical nephroureterectomy in 71 patients with NOM0 renal pelvic cancer.

with WBC counts $\geq 7600 / \mu$ l than in those with WBC counts $<7600 / \mu 1(\mathrm{P}=0.0243$; data not shown).

The Cox proportional hazards model was used to evaluate preoperative predictors of EURFS. Univariate analysis showed cT $\geq 3$, WBC count $\geq 7600 / \mu 1$, neutrophils $\geq 4,500 / \mu 1$, and LDH $\geq 210$ IU/1 significantly associate with EURFS. Multivariate analysis showed that $\mathrm{cT} \geq 3(\mathrm{HR}=3.759)$ and $\mathrm{LDH} \geq 210(\mathrm{HR}=3.521)$ were significant predictors of EURFS, but WBC counts $\geq 7600 / \mu 1$ and neutrophil counts $\geq 4,500 / \mu 1$ were not (Table II).
The 71 patients were stratified into three groups according to the number of risk factors $(n=0,1$, or 2$)$. The 3 -year EURFS rates of the three groups were 94.5, 76.3 and $33.3 \%$, respectively (Fig. 3A). We found a significant difference in EURFS rates between the low-risk and intermediate-risk groups $(\mathrm{P}=0.0353)$ and the difference was close to significant between the intermediate-risk and high-risk groups $(\mathrm{P}=0.069)$. When the postoperative factors in each group were further evaluated, the high-risk group was associated with higher pT stages and higher percentages of LVI (Table III).

\section{Discussion}

The present study focused on RPC and evaluated preoperative factors to identify predictors of EUR. Multivariate analysis revealed that $\mathrm{cT}$ stage $\geq 3$ and preoperative $\mathrm{LDH}$ level were independent predictors of EUR. The present study also stratified the patients into three groups according to the number of risk factors. In our previous study evaluating UTC, we identified $\mathrm{cT} \geq 3$, tumor length, positive urine cytology, and eGFR $<60$ as independent predictors for EUR (11). Only cT stage was an independent predictor for both UTC and RPC. Also, in our previous study, univariate analysis revealed that cT stage, tumor length along the ureter, maximal tumor diameter, positive urine cytology, NLR $\geq 3.0$, and eGFR $\leq 60 \mathrm{ml} / \mathrm{min} / 1.73 \mathrm{~m}^{2}$ were significant risk factors for UTC (11). Notably, even significant factors identified by univariate analysis considerably differed between patients with UTC and those with RPC. Because preoperative predictors for EUR 
Table II. Preoperative factors predicting extra-urothelial recurrence in NOM0 patients with renal pelvic cancer.

\begin{tabular}{|c|c|c|c|c|}
\hline \multirow[b]{2}{*}{ Variables } & \multirow{2}{*}{$\begin{array}{l}\text { Univariate } \\
\text { P-value }\end{array}$} & \multicolumn{3}{|c|}{ Multivariate } \\
\hline & & P-value & $\begin{array}{l}\text { Hazard } \\
\text { ratio }\end{array}$ & $\begin{array}{l}\text { Relative risk } \\
\text { ratio } 95 \% \mathrm{CI}\end{array}$ \\
\hline Age $\geq 65 \mathrm{y} / \mathrm{o}$ & 0.2844 & & & \\
\hline Gender & 0.7859 & & & \\
\hline Tumor side & 0.7167 & & & \\
\hline Past history of BT and/or concomitant BT & 0.2455 & & & \\
\hline Symptomatic & 0.3664 & & & \\
\hline Concomitent ureteral lesion (radiological) & 0.6902 & & & \\
\hline Presence of hydrocalyx & 0.4242 & & & \\
\hline Clinical T stage $\geq 3$ & 0.0143 & 0.0244 & 3.759 & $1.188-11.905$ \\
\hline Maximal tumor size $\geq 3 \mathrm{~cm}$ & 0.6033 & & & \\
\hline Positive urine cytology & 0.1418 & & & \\
\hline $\mathrm{WBC} \geq 7600 / \mu 1$ & 0.0375 & & & \\
\hline Neutrophil $\geq 4,500 / \mu 1$ & 0.0281 & & & \\
\hline $\mathrm{CRP} \geq 0.3 \mathrm{mg} / \mathrm{dl}$ & 0.4225 & & & \\
\hline $\mathrm{NLR} \geq 2.0$ & 0.07 & & & \\
\hline $\mathrm{LDH} \geq 210 \mathrm{IU} / 1$ & 0.019 & 0.0322 & 3.521 & $1.112-11.111$ \\
\hline $\mathrm{eGFR}<60 \mathrm{ml} / \mathrm{min} / 1.73 \mathrm{~m}^{2}$ & 0.9113 & & & \\
\hline
\end{tabular}

BT, bladder tumor; CRP, C-reactive protein; eGFR, estimated glomerular filtration ratio; LDH, lactate dehydrogenase; NLR, neutrophil-to-lymphocyte rate; WBC, white blood cell; CI, confidence interval.

Table III. Distribution on postoperative prognostic factors and extraurothelial recurrence in each risk group.

\begin{tabular}{lcccr}
\hline Variables & $\begin{array}{c}\text { Low-risk } \\
(\mathrm{n}=39)\end{array}$ & $\begin{array}{c}\text { Intermediate-risk } \\
(\mathrm{n}=26)\end{array}$ & $\begin{array}{c}\text { High-risk } \\
(\mathrm{n}=6)\end{array}$ & P-value \\
\hline $\begin{array}{l}\text { Pathological T (pT) stage } \\
\text { (pTis/a/1/2/3/4) }\end{array}$ & $2 / 12 / 7 / 3 / 15 / 0$ & $0 / 5 / 1 / 2 / 18 / 0$ & $0 / 0 / 0 / 1 / 4 / 1$ & $0.0150^{\mathrm{a}}$ \\
pT stage $<2$ vs. $\geq 2$ & $21 / 18$ & $6 / 20$ & $0 / 6$ & $0.0058^{\mathrm{a}}$ \\
Grade (grade 1/2/3) & $1 / 15 / 23$ & $1 / 6 / 19$ & $0 / 0 / 6$ & $0.2936^{\mathrm{a}}$ \\
LVI (-/+) & $32 / 7$ & $17 / 9$ & $2 / 4$ & $0.0311^{\mathrm{a}}$ \\
Adjuvant chemotherapy (-/+) & $36 / 3$ & $20 / 6$ & $3 / 3$ & $0.0208^{\mathrm{a}}$ \\
Lymph node sampling (-/+) & $32 / 7$ & $22 / 4$ & $4 / 2^{\mathrm{b}}$ & 0.5173 \\
Extraurothelial recurrence (-/+) & $37 / 2^{\mathrm{c}}$ & $20 / 6^{\mathrm{d}}$ & $2 / 4^{\mathrm{e}}$ & $0.0005^{\mathrm{a}}$ \\
\hline
\end{tabular}

DM, distant metastasis; LNM, lymph node metastasis; LVI, lymphovascular invasion. ${ }^{a}$ Analysed by chi-square test, ${ }^{b} \mathrm{LNM}$ was present in 1 patient (pt), ${ }^{\mathrm{C}} \mathrm{LNM}+\mathrm{DM}, 1$ pt; DM, 1 pt. ${ }^{\mathrm{d}} \mathrm{LNM}$ only, 1 pt., DM only, 2 pts., LNM+DM, 3 pts., ${ }^{\mathrm{e}} \mathrm{LNM}$ only, 2 pts., DM only, 2 pts.

differed (with the exception of cT stage between UTC and $\mathrm{RPC}$ ), it may be more advantageous to determine preoperative predictors for EUR in patients with UTC and those with RPC, separately.

Previously, a number of authors attempted to identify preoperative predictors of UTUC recurrence (3-5). Hashimoto et al (5) identified that cT stage and neutrophil count of $\geq 4,000 / \mu 1$ were independent predictors of recurrence (5). However, the present study separately evaluated patients with UTC and those with RPC, which was a novel approach. Another three studies reported that ureteroscopic grade and tumor architecture determined by ureteroscopy were independent predictors of muscle-invasive or non-organ-confined disease (2-4). Although factors which can be determined by ureteroscopic examination such as ureteroscopic grade and tumor architecture appear to be strong predictors for EUR, the current study aimed to identify predictors for EUR by using simple preoperative factors that can be determined without ureteroscopic examination.

The results of the present study demonstrated that cT stage was an independent predictor for EUR. It is difficult to determine pathological $\mathrm{T}$ stage by radiological examination. By 
A

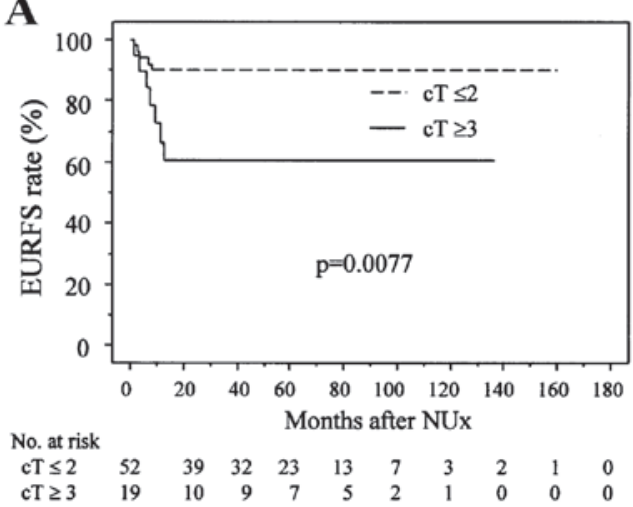

C

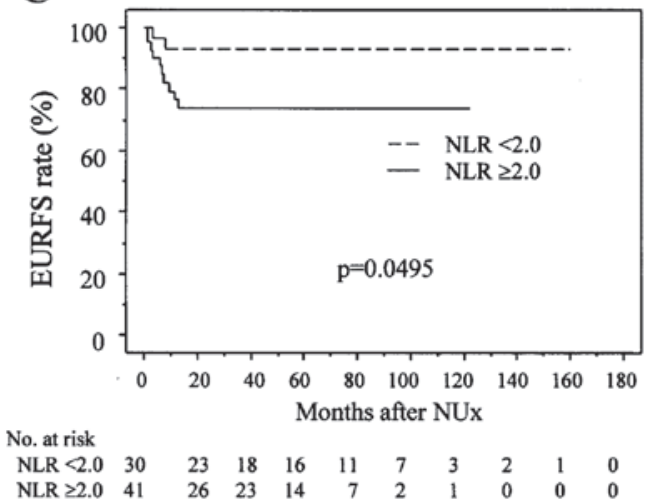

B

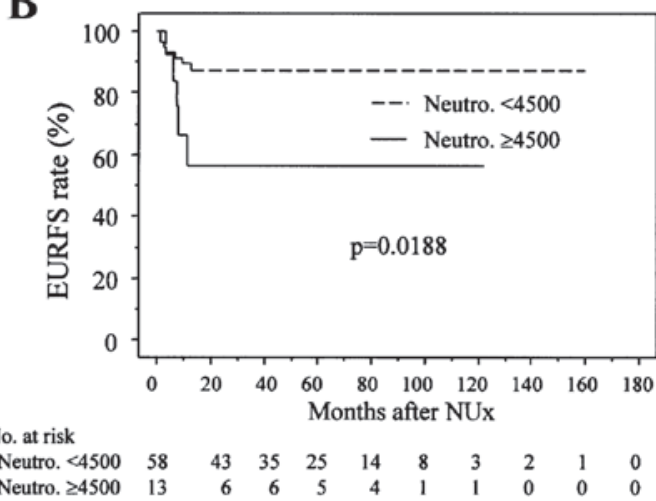

D

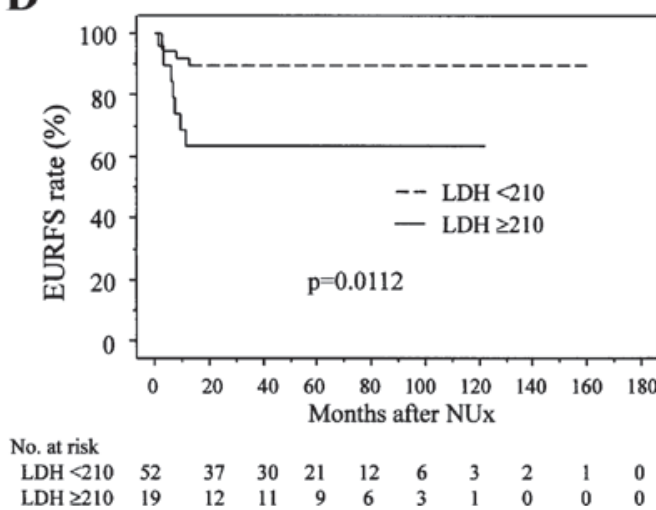

Figure 2. Associations between preoperative factors and EUR. Kaplan-Meier analysis revealed (A) cT $\geq 3$, (B) neutrophil count $\geq 4,500 / \mu 1$, (C) NLR $\geq 2.0$, and (D) $\mathrm{LDH} \geq 210 \mathrm{IU} / 1$, were correlated with a greater risk of EUR $(\mathrm{P}<0.05)$. EUR, extraurothelial recurrence.

A

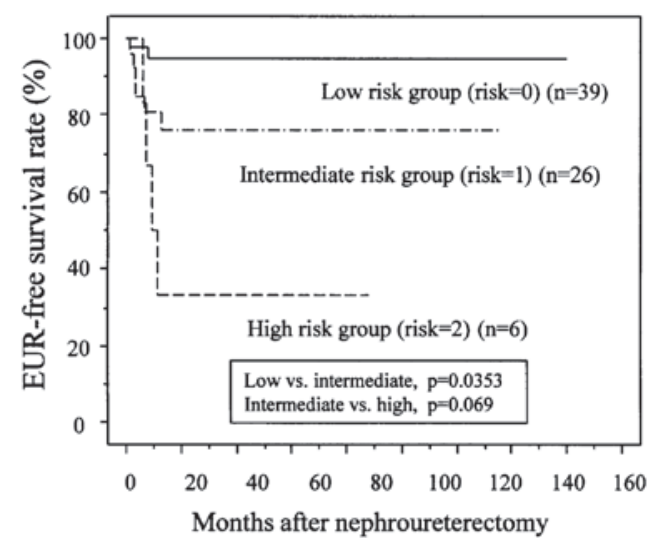

B

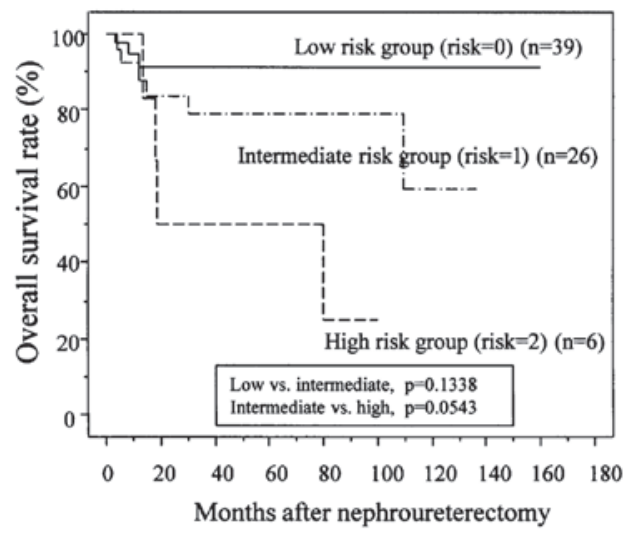

No. at risk

$\begin{array}{clllllllllll}\text { Risk }=0 & 39 & 29 & 22 & 16 & 9 & 4 & 2 & 2 & 1 & 0\end{array}$

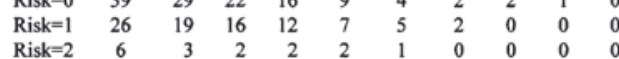

Figure 3. Risk stratification using two predictive factors for EUR. (A) Risk stratification according to the number of risk factors. Patients stratified into low-, intermediate-, and high-risk groups (0, 1, and 2 risk factors, respectively), had 1- and 3-year EURFS rates of 94.5 and 94.5\%, 80.6 and 76.3\%, and 33.3 and 33.3\%, respectively. Kaplan-Meier EURFS curves show a significant difference between the low- and intermediate-risk groups (P=0.0353) and closely significant differences between the intermediate- and high-risk groups $(\mathrm{P}=0.059)$. (B) OS rates in each risk group. Patients stratified into low-, intermediate-, and high-risk groups, had 1- and 3-year OS rates of 94.6 and 91.5\%, 92.3 and $79.1 \%$, and 100 and 50\%, respectively. Patients in the low-risk group lived longer than those in the intermediate-risk group $(\mathrm{P}=0.1338)$, and patients in the intermediate-risk group lived longer than those in the high-risk group $(\mathrm{P}=0.0543)$.

radiography, microscopic $\mathrm{T} 3$ tumors do not show obvious extension towards renal parenchyma or peripelvic fat tissue, and are likely to be categorized as $\mathrm{cT} \leq 2$. In the present study, a total of 17 of 19 tumors which were categorized as $\mathrm{cT} \geq 3$ were diagnosed as pT3 or more $(89.5 \%, 16$ patients had pT3 tumors and one had pT4). By contrast, 21 of the 51 tumors categorized as cT $\leq 2$ were diagnosed as pT3 (41.2\%), suggesting there was difficulty in determining the $\mathrm{pT}$ stages by radiological examinations. Furthermore, pT3 tumors categorized as $\mathrm{cT} \leq 2$ appeared to have a better EURFS rate than pT3 tumors that were 
categorized as cT $\geq 3$ (2-year-EURFS rate: 85.2 vs. $59.6 \%$, $\mathrm{P}=0.1381)$. This may be one of the reasons for $\mathrm{cT}$ stage being a strong predictor for EUR.

Preoperative LDH level is a novel preoperative predictor for EUR in renal pelvic cancer patients. Additionally, LDH level is reported to be an important prognostic factor in patients with metastatic renal cell carcinoma (14). LDH level likely reflects the quantity of tumor cells in the body. Therefore, elevated LDH levels may reflect the presence of latent metastases in patients with radiological NOM0 RPC. Preoperative LDH levels appeared to be higher in RPC patients with EUR $(n=12)$ compared with those who did not recur $(n=59$; 199 vs. $181 \mathrm{IU} / \mathrm{L}, \mathrm{P}=0.0591$ by Mann-Whitney U test). When 12 patients who had EUR were reviewed, LDH once decreased postoperatively in all 12 patients. When EURs were detected, only 3 patients had LDH levels $\geq 210 \mathrm{IU} / 1$. However, in 9 of the 12 patients $(75 \%)$ maximal LDH levels after EUR were more than $210 \mathrm{IU} / 1$ (215-5370). Although postoperative LDH levels appeared not to correlate with systemic quantity of tumor cells in all patients, postoperative LDH levels increased in the majority of the 12 patients as their disease was progressed.

In previous reports evaluating prognostic factors after RNU, inflammatory indices, such as CRP $(15,16)$, neutrophil count (5) and NLR (17), were independent prognostic factors. Saito et al (15) reported that preoperative CRP level, pT stage, and lymph node involvement were significant prognostic factors for disease-specific and recurrence-free survival. A previous multi-institutional study revealed that elevated preoperative NLR was an independent predictor for disease recurrence (17). However, these inflammatory indices were not independent factors in the present study or our previous study evaluating UTC (11). A possible reason to explain the differences between the findings of our two studies and previous studies $(15,17)$ may be that we evaluated RPC and UTC separately. Another possible reason may be that we evaluated only NOM0 UTUC patients.

In addition, the present study did not evaluate tumor markers, such as cancer antigen 19-9, carcinoembryonic antigen, or squamous cell carcinoma antigen, in the present study. These factors were evaluated in our previous study of UTC (11). However, these markers were examined for only $60-70 \%$ of patients with RPC. When these markers were analyzed by univariate analyses, none were significant (data not shown).

In our previous study of UTC, eGFR was an independent predictor for EUR (11). However, eGFR was not an independent predictor for EUR in the current study. In case of RPC, the whole dilation of renal pelvis and renal calyces in the unilateral kidney can occur only when a tumor is located near the ureteropelvic junction or when the renal pelvis contains a large tumor. If the tumor is located near the renal calyx, the whole dilation of renal pelvis and renal calyces is not likely to occur even if the tumor is invasive. Because tumors located within limited renal calyces that invade the renal parenchyma usually decreases renal function partially, the impairment of renal function is probably minimal compared to that in patients with tumors that cause the whole dilation of renal pelvis and renal calyces. By contrast, invasive UTC can easily cause the whole dilation of renal pelvis and renal calyces, and eGFR can decrease markedly. In our previous study (11), the median
eGFR of 70 patients with UTC was $58.9 \mathrm{ml} / \mathrm{min} / 1.73 \mathrm{~m}^{2}$ and in the present study this was $64.8 \mathrm{ml} / \mathrm{min} / 1.73 \mathrm{~m}^{2}$ (UTC vs. RPC, $\mathrm{P}=0.0722$ by the Mann-Whitney $\mathrm{U}$ test). Moreover, although 56 of 70 patients with UTC exhibited varied degrees of hydronephrosis, 26 of 71 patients with RPC had selected hydrocalyx or hydronephrosis. The presence of hydronephrosis is related to tumor invasiveness in UTC (18), thus eGFR might be an independent predictor for EUR in patients with UTC in our previous study (11). By contrast, partial RPC invasion of the renal parenchyma (pT3 disease) is likely to slightly decrease eGFR. In the present study, eGFR was not a significant factor to predict EUR even by univariate analysis.

In addition to EUR prediction, our risk stratification using preoperative factors also appeared to predict patient survival (Fig. 3B), which was nicely correlated with $\mathrm{pT}$ stage and the presence of LVI (Table III). As shown in Fig. 3, low-risk patients were at a relatively low risk for EUR (3-year EURFS=94.5\%) and may be candidates for RNU without LND. In this study, there were 39 (54.9\%) low-risk patients and LND may be potentially avoided in more than half of NOM0 patients with RPC, according to the proposed risk classification. By contrast, high-risk patients experience rapid recurrence within a short period after RNU. 50\% of the high-risk patients who developed recurrence had distant metastases without LNM at the initial radiologically confirmed recurrence. Therefore, neoadjuvant chemotherapy should be considered for high-risk patients and following RNU with ELND may improve survival of some high-risk patients. In two multicenter retrospective studies, neoadjuvant chemotherapy followed by RNU was suggested to improve the prognosis of UTUC patients with LNM $(19,20)$. Furthermore, a recent meta-analysis suggested that cisplatin-based neoadjuvant chemotherapy might prolong survival of UTUC patients (21).

The present study has several limitations that should be addressed. First, it was a nonrandomized, retrospective, single-center study; therefore, external validation and further prospective studies with larger numbers of patients are required. Second, some patients underwent LND, the extent of which was not standardized. Analysis of more uniform populations, such as patients who did not undergo LND, presents a better method to determine EUR. However, 58 (81.7\%) of 71 patients did not undergo LND, limited LND (removal of $\mathrm{LN}$ around renal hilus) was performed in 11 patients (15.5\%), and ELND was performed for only two patients (these patients did not have LNM pathologically). Of the 39 low-risk patients, only 7 (17.9\%) underwent LND and none had pathological LNM. Therefore, LND appeared to be able to be omitted in the majority of low-risk patients. Third, 12 patients with pathologically confirmed muscle-invasive RPC received postoperative chemotherapy. However, 3 (25\%) of 12 patients who underwent postoperative chemotherapy developed recurrence, as compared to only $9(15.5 \%)$ of 59 patients who did not. There was no apparent benefit of postoperative chemotherapy on EUR in our series. Moreover, only 3 (7.7\%) patients in the low-risk group received postoperative chemotherapy (Table III) and the effect of chemotherapy on recurrence in the low-risk patients appeared to be minimal. By contrast, 23.1\% of the intermediate-risk patients and $50 \%$ of the high-risk patients received postoperative chemotherapy. Fourth, EURFS rate was rather better compared to some previous reports $(2,3)$. 
A small number of RPC patients in a single institute might influence on the oncological outcome in the present study. Furthermore, adjuvant chemotherapy may somewhat influence patient outcome. Although the present study had several limitations, it was possible to establish a risk classification for EUR in RPC patients using preoperative factors that could be easily determined.

In conclusion, clinical $\mathrm{T}$ stage $\geq 3$ and $\mathrm{LDH} \geq 210 \mathrm{IU} / 1$ were independent preoperative predictors of EUR in patients with N0M0 RPC treated by RNU. Risk stratification using these preoperative factors may be useful to select candidates for neoadjuvant chemotherapy and for RNU without LND. However, further studies with larger numbers of patients and external validation are required.

\section{Acknowledgements}

The authors thank Professor Jun Nakashima, Tokyo Medical University, for his helpful comments and valuable discussion.

\section{References}

1. Saito K, Kawakami S, Fujii Y, Sakura M, Masuda $\mathrm{H}$ and Kihara K: Lymphovascular invasion is independently associated with poor prognosis in patients with localized upper urinary tract urothelial carcinoma treated surgically. J Urol 178: 2291-2296; discussion 2296, 2007.

2. Kikuchi E, Margulis V, Karakiewicz PI, Roscigno M, Mikami S, Lotan Y, Remzi M, Bolenz C, Langner C, Weizer A, et al: Lymphovascular invasion predicts clinical outcomes in patients with node-negative upper tract urothelial carcinoma. J Clin Oncol 27: 612-618, 2009.

3. Margulis V, Shariat SF, Matin SF, Kamat AM, Zigeuner R, Kikuchi E, Lotan Y, Weizer A, Raman JD and Wood CG; Upper Tract Urothelial Carcinoma Collaboration. The Upper Tract Urothelial Carcinoma Collaboration: Outcomes of radical nephroureterectomy: A series from the upper tract urothelial carcinoma collaboration. Cancer 115: 1224-1233, 2009.

4. Zigeuner R, Shariat SF, Margulis V, Karakiewicz PI, Roscigno M, Weizer A, Kikuchi E, Remzi M, Raman JD, Bolenz C, et al: Tumour necrosis is an indicator of aggressive biology in patients with urothelial carcinoma of the upper urinary tract. Eur Urol 57: 575-581, 2010

5. Hashimoto T, Ohno Y, Nakashima J, Gondo T, Ohori M and Tachibana M: Clinical significance of preoperative peripheral blood neutrophil count in patients with non-metastatic upper urinary tract carcinoma. World J Urol 31: 953-958, 2013.

6. Brien JC, Shariat SF, Herman MP, Ng CK, Scherr DS, Scoll B, Uzzo RG, Wille M, Eggener SE, Terrell JD, et al: Preoperative hydronephrosis, ureteroscopic biopsy grade and urinary cytology can improve prediction of advanced upper tract urothelial carcinoma. J Urol 184: 69-73, 2010.

7. Margulis V, Youssef RF, Karakiewicz PI, Lotan Y, Wood CG, Zigeuner R, Kikuchi E, Weizer A, Raman JD, Remzi M, et al: Preoperative multivariable prognostic model for prediction of nonorgan confined urothelial carcinoma of the upper urinary tract. J Urol 184: 453-458, 2010.
8. Favaretto RL, Shariat SF, Savage C, Godoy G, Chade DC, Kaag M, Bochner BH, Coleman J and Dalbagni G: Combining imaging and ureteroscopy variables in a preoperative multivariable model for prediction of muscle-invasive and non-organ confined disease in patients with upper tract urothelial carcinoma. BJU Int 109: 77-82, 2012.

9. Park S, Hong B, Kim CS and Ahn H: The impact of tumor location on prognosis of transitional cell carcinoma of the upper urinary tract. J Urol 171: 621-625, 2004.

10. Akdogan B, Dogan HS, Eskicorapci SY, Sahin A, Erkan I and Ozen H: Prognostic significance of bladder tumor history and tumor location in upper tract transitional cell carcinoma. J Urol 176: 48-52, 2006.

11. Ito K, Kuroda K, Asakuma J, Hamada S, Tachi K, Tasaki S, Sato A, Horiguchi A, Seguchi K and Asano T: Preoperative risk factors for extraurothelial recurrence in patients with ureteral cancer treated with radical nephroureterectomy. J Urol 191: $1685-1692,2014$

12. Sobin LH, Gospodarowicz M and Wittekind C: Kidney. In: TNM classification of malignant tumors. 7th edition. Wiley-Blackwell, New York, NY: p258-p261, 2009.

13. Ohno Y, Nakashima J, Ohori M, Hatano T and Tachibana M: Pretreatment neutrophil-to-lymphocyte ratio as an independent predictor of recurrence in patients with nonmetastatic renal cell carcinoma. J Urol 184: 873-878, 2010.

14. Motzer RJ, Mazumdar M, Bacik J, Berg W, Amsterdam A and Ferrara J: Survival and prognostic stratification of 670 patients with advanced renal cell carcinoma. J Clin Oncol 17: 2530-2540, 1999.

15. Saito K, Kawakami S, Ohtsuka Y, Fujii Y, Masuda H, Kumagai J, Kobayashi T, Kageyama Y and Kihara K: The impact of preoperative serum $\mathrm{C}$-reactive protein on the prognosis of patients with upper urinary tract urothelial carcinoma treated surgically. BJU Int 100: 269-273, 2007.

16. Tanaka N, Kikuchi E, Shirotake S, Kanao K, Matsumoto K, Kobayashi H, Miyazaki Y, Ide H, Obata J, Hoshino K, et al: The predictive value of C-reactive protein for prognosis in patients with upper tract urothelial carcinoma treated with radical nephroureterectomy: A multi-institutional study. Eur Urol 65: 227-234, 2014.

17. Tanaka N, Kikuchi E, Kanao K, Matsumoto K, Shirotake S, Miyazaki Y, Kobayashi H, Kaneko G, Hagiwara M, Ide H, et al: A multi-institutional validation of the prognostic value of the neutrophil-to-lymphocyte ratio for upper tract urothelial carcinoma treated with radical nephroureterectomy. Ann Surg Oncol 21: 4041-4048, 2014

18. Ito Y, Kikuchi E, Tanaka N, Miyajima A, Mikami S, Jinzaki M and Oya M: Preoperative hydronephrosis grade independently predicts worse pathological outcomes in patients undergoing nephroureterectomy for upper tract urothelial carcinoma. J Urol 185: 1621-1626, 2011.

19. Youssef RF, Shariat SF, Lotan Y, Wood CG, Sagalowsky AI, Zigeuner R, Kikuchi E, Weizer A, Raman JD, Remzi M, et al: Upper urinary tract urothelial carcinoma with loco-regional nodal metastases: Insights from the upper tract urothelial carcinoma collaboration. bju int 108: 1286-1291, 2011.

20. Kitamura H, Igarashi M, Tanaka T, Shindo T, Masumori N, Tamakawa M, Kawaai Y and Tsukamoto T: A role for preoperative systemic chemotherapy in node-positive upper tract urothelial carcinoma treated with radical nephroureterectomy. Jpn J Clin Oncol 42: 1192-1196, 2012.

21. Leow JJ, Martin-Doyle W, Fay AP, Choueiri TK, Chang SL and Bellmunt J: A systematic review and meta-analysis of adjuvant and neoadjuvant chemotherapy for upper tract urothelial carcinoma. Eur Urol 66: 529-541, 2014. 